\title{
Frequency-Comb Regeneration for Self-Homodyne Superchannels
}

Abel Lorences-Riesgo, Tobias A. Eriksson, Attila Fülöp, Magnus Karlsson and Peter A. Andrekson

Department of Microtechnology and Nanoscience, Chalmers University of Technology, SE-41296 Gothenburg, Sweden. lorences@chalmers.se

Abstract We demonstrate frequency-comb regeneration from two received unmodulated carriers using a parametric mixer. For $10 \mathrm{~dB}$ optical signal-to-noise ratio of the unmodulated carriers, up to 60 carriers are generated without linewidth degradation.

\section{Introduction}

The coherent receiver in combination with digital signal processing (DSP) has opened the possibility to use spectrally efficient modulation formats such as higher order quadrature amplitude modulation (QAM). When the number of signalling levels is increased, the requirements on the linewidth of the transmitter as well as receiver lasers are also increased ${ }^{11}$. The self-homodyne (SH) detection technique ${ }^{2}$, where an unmodulated copy of the transmitter carrier is co-propagated through the transmission fiber and used as a local oscillator (LO), has been shown to greatly reduce the linewidth requirement. The drawback of $\mathrm{SH}$ detection is that the spectral efficiency (SE) is reduced, often by $50 \%$ since the carrier is transmitted in the orthogonal polarization to the signal. A similar technique is to transmit a frequency shifted carrier, originating from the transmission laser. This technique in combination with an optical phase-locked loop has successfully been used to detect QAM signals of up to 2048-QAM ${ }^{3}$.

Optical frequency combs can be used on the transmitter side to create many wavelengthdivision multiplexing (WDM) carriers from a single laser source. Recently, the idea of regenerating an identical frequency comb at the receiver was suggested ${ }^{4}$. This technique can be used to achieve self-homodyne superchannel receivers while sacrificing a tolerable amount of SE since only one or two carrier tones are transmitted. For this purpose, the comb needs to be regenerated with high fidelity from the carriers which are degraded during the transmission.

In this paper, we demonstrate generation of up to 71 carriers from two noisy center carriers using a parametric mixer. The use of optical injection locking (OIL) enables frequency-comb regeneration when the received carriers are impaired by noise. Linewidth measurements confirm that the generated carriers are not degraded by phase

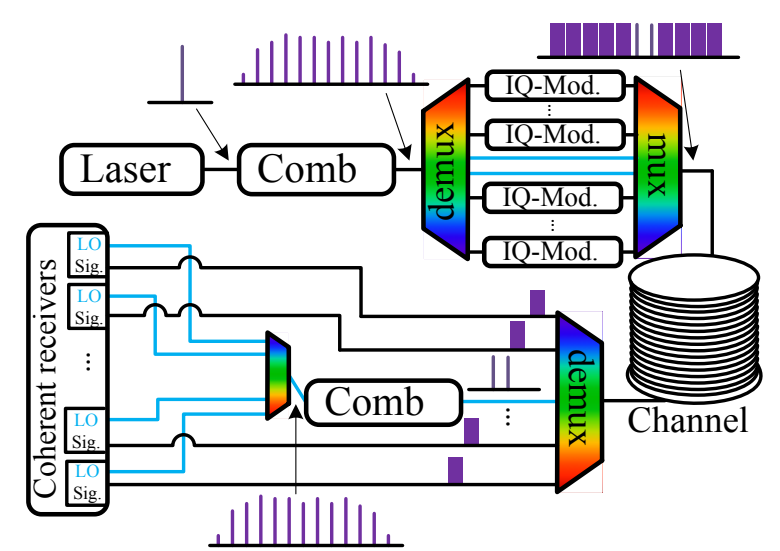

Fig. 1: Schematics of a single-source SH superchannel

noise for $15 \mathrm{~dB}$ optical-to-signal ratio (OSNR) of the two input carriers. At $10 \mathrm{~dB}$ OSNR, up to 60 carriers can be generated without major linewidth degradation.

\section{Single-Source Self-Homodyne Superchannel}

The general idea of a single-source $\mathrm{SH}$ superchannel is shown in Fig. 1. In the transmitter, a single laser is used as source to a comb generator. All carriers are modulated with polarizationmultiplexed (PM) signals of choice, except the two center tones which are left unmodulated. The signals and the unmodulated carriers are then combined and transmitted over the link. In the receiver, the two unmodulated carriers are filtered out and sent to a second frequency comb. This comb generates carriers which can be used for homodyne detection of the whole super-channel and reduce the complexity of the DSP for higherorder modulation formats. The carriers must be regenerated carriers without being degraded by amplitude or phase noises even though the received carrier tones are severely distorted by noise at the receiver input. In this paper, we demonstrate that this is possible by using OIL and a parametric comb (frequency comb generated by the Kerr effect). It is important to realize that linewidth preservation in the parametric fre- 


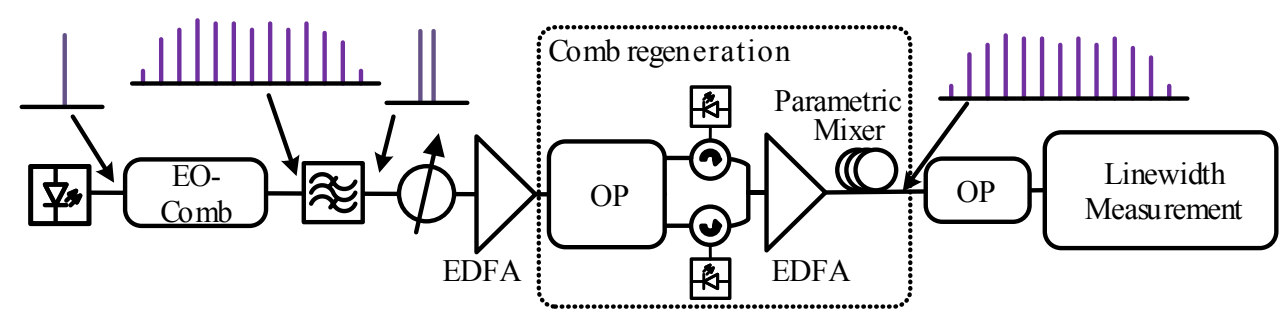

Fig. 2: Experimental setup for evaluation of the comb regeneration performance with regard to the input OSNR. Acronyms are explained within the text.

quency comb is possible since the two carriers which seed the parametric frequency comb are frequency-locked ${ }^{5}$.

\section{Experimental Setup}

The experimental setup shown in Fig. 2 was implemented in order to analyze the requirements for frequency-comb regeneration with highfidelity. A laser centered at $1553.3 \mathrm{~nm}$ and linewidth of about $175 \mathrm{kHz}$ fed an electro-optic comb formed by a Mach-Zehnder modulator and two phase modulators. As shown in Fig. 3(a), 62 carriers spaced by $25 \mathrm{GHz}$ were generated in a $20 \mathrm{~dB}$ bandwidth. The carriers at $1553.3 \mathrm{~nm}$ and $1553.5 \mathrm{~nm}$ were filtered and attenuated before being injected into an erbium-doped amplifier (EDFA) which served as noise-loading stage. After this EDFA, the carriers were directed to two different paths by the use of an optical processor. Apart from splitting the carriers, the optical processor allowed us to control the power of each carrier. In each path, an OIL stage removed noise from the carriers while simultaneously amplifying them. Then, both carriers were combined before being injected into a high-power EDFA. After the EDFA, the cascade of a highly-nonlinear fiber (HNLF), single-mode fiber (SMF) and HNLF allowed the generation of multiple carriers. After the parametric comb, we filtered out every carrier and measured its linewidth. The linewidth was measured by splitting the carrier in two path with about $27 \mathrm{~km}$ difference. In one path, we included an acoustic-optic modulator that shifted the frequency by $27 \mathrm{MHz}$. The two paths were combined and detected by a photodiode. The electrical signal was finally analyzed in an electrical spectrum analyzer.

\section{Results and Discussion}

In order to confirm the importance of the OIL stage, we show the spectra of the parametric comb for the cases with and without this stage in Fig. 3(b). If the OIL stage is not included, we observed that the generated comb is limited by the OSNR despite the high OSNR per carrier $(22 \mathrm{~dB})$ at the input of the comb regeneration stage. This OSNR degradation is due to two factors: the noise is amplified in the HNLFs and the power of the carriers is distributed in order to generate new carriers. When having the OIL stage, the parametric comb generated up to 71 carriers in a $20 \mathrm{~dB}$ bandwidth which much higher OSNR than in the case of not including the OIL stage. The OSNR of the generated carriers could not be measured accurately in an optical-spectrum analyzer due to the low spacing between carriers.

The 3-dB linewidth of the carriers generated by the parametric comb was measured for two different input OSNRs, $10 \mathrm{~dB}$ and $15 \mathrm{~dB}$, measured at the input of the com regeneration stage. Moreover, we also assessed the importance of the path difference between the two pumps in the OIL stage in the case of $15 \mathrm{~dB}$ OSNR. Our measurements included the case of path difference of $2 \mathrm{~m}, 30 \mathrm{~cm}$ and path matched within $\mathrm{mm}$ scale. The linewidth results are shown in Fig. 3(c) in which the carrier number is counted taking as reference the laser wavelength. When the input OSNR is $15 \mathrm{~dB}$ and the OIL is path matched within tens of $\mathrm{cm}$ scale, no linewidth degradation is observed. However, if the path difference is about $2 \mathrm{~m}$, we can observe that the carriers are degraded and we could not estimate the linewidth beyond about the $\pm 20^{\text {th }}$ carriers. When the input OSNR is $10 \mathrm{~dB}$, we can also observe that the linewidth of the edge carriers is degraded and only the linewidth of about 62 carriers could be estimated with this OSNR. With such low OSNR, the power injected into the slave lasers must be thoroughly optimized since it determines the bandwidth of the IL, and therefore the phase-noise penalty caused by the amplified spontaneous emission (ASE) noise. The small difference between the cases of $10 \mathrm{~dB}$ and $15 \mathrm{~dB}$ OSNR per carrier can be due to the fact that at $15 \mathrm{~dB}$ OSNR, the OIL stage was not perfectly optimized due to the large range of operation.

It is important to realize that linewidth preservation indicates that the carriers are frequency and 

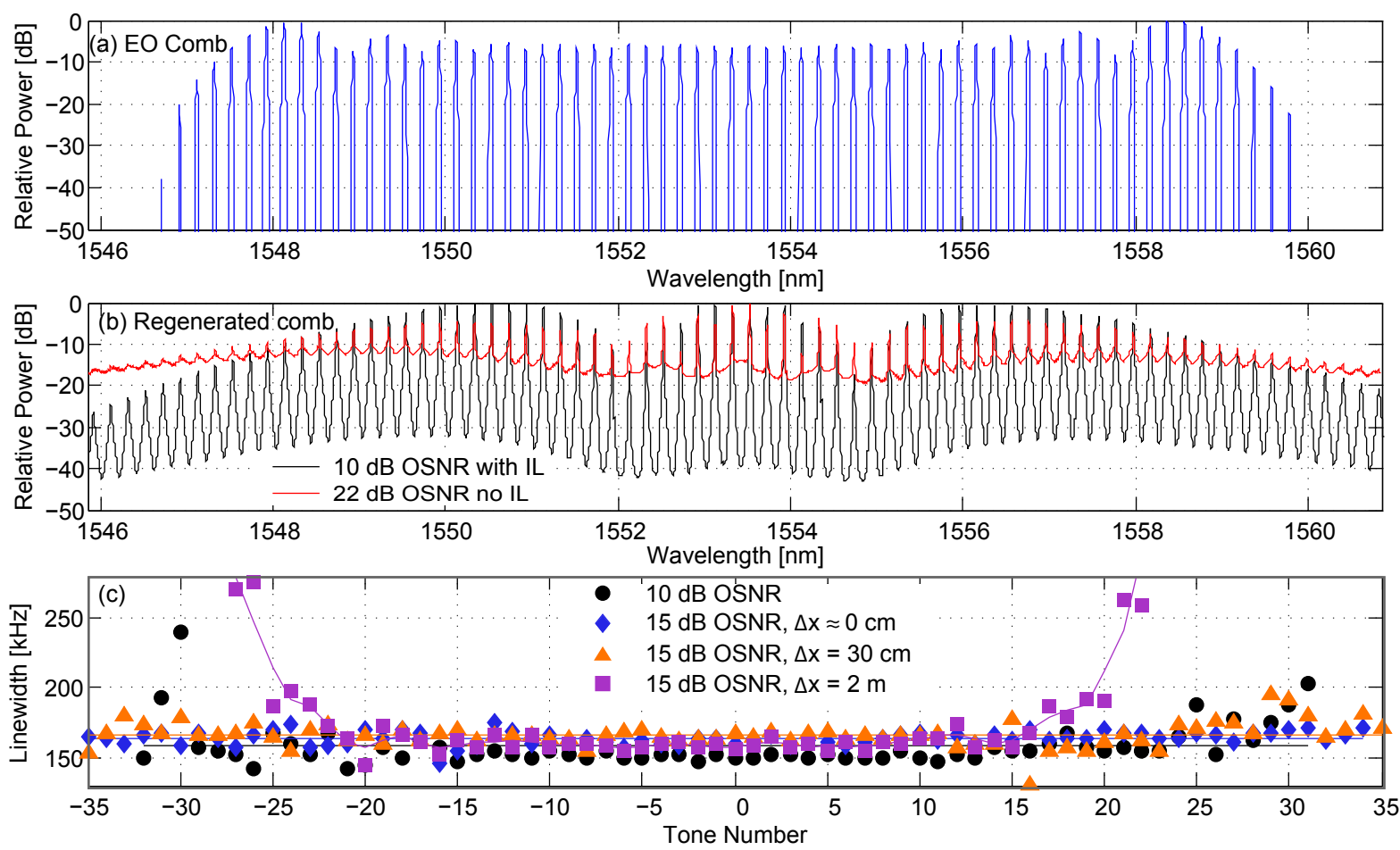

Fig. 3: (a) Electro-optic frequency comb with $25 \mathrm{GHz}$ spacing, (b) regenerated parametric frequency comb with $25 \mathrm{GHz}$ spacing and (c) linewidth of the regenerated frequency comb for different input conditions

phase-locked. If the carriers are not frequency and phase locked, the linewidth do scale with the number of tone in a relation between linear and quadratic ${ }^{5}$. Therefore, when the linewidth is preserved, the regenerated carriers are frequencylocked to the central carriers in the same way as in the transmitter comb.

A received OSNR as low as $10 \mathrm{~dB}$ would be sufficient to receive a superchannel consisting of Nyquist-spaced 25 Gbaud PM-quadrature phaseshift keying (QPSK) signals, as the theoretical required OSNR is around $9 \mathrm{~dB}$ for a bit error-rate (BER) of $2 \times 10^{-2}$ not accounting for implementation penalties. Further, the two pilot carriers could be transmitted with higher launch power than the data channels if higher OSNR is required in the frequency-comb regeneration stage. In cases of higher-order QAM signals for which the proposed scheme is designed, the required OSNR in the data channels would be sufficient to regenerate a frequency comb without linewidth degradation.

In a transmission system, the carriers are influence by nonlinear crosstalk from the data into the carriers as well as additive noise. However, higher-order QAM signals which are expected to be used in combination with the proposed idea do not show major tolerance towards signal-carrier nonlinear crosstalk. Therefore, we do not expect major penalty from the nonlinear crosstalk in these cases.

\section{Conclusions}

We have demonstrated frequency-comb regeneration without linewidth degradation by using an OIL stage followed by a parametric comb. At $10 \mathrm{~dB}$ input OSNR, up to 60 carriers were generated which might be used as LOs in a singlesource SH superchannel scheme. Only two unmodulated carriers are necessary for this comb generation which leads to an spectral overhead of about $4 \%$.

\section{Acknowledgements}

This work is supported by the European Research Council under grant agreement ERC2011-AdG-291618 PSOPA. The authors thank OFS Denmark for providing HNLFs.

\section{References}

[1] T. Pfau et al., "Hardware-Efficient Coherent Digital Receiver Concept With Feedforward Carrier Recovery for $M$-QAM Constellations," J. Lightwave Technol., vol. 27, p. 989 (2009).

[2] T. Miyazaki and F. Kubota, "PSK Self-Homodyne Detection Using a Pilot Carrier for Multibit/Symbol Transmission with Inverse-RZ Signal," Photon. Technol. Lett., 17, p. 1334 (2005).

[3] S. Beppu, et al., "2048 QAM (66 Gbit/s) Single-carrier Coherent Optical Transmission Over $150 \mathrm{~km}$ with a Potential SE of 15.3 bit/s/Hz," Opt. Exp., 23, p. 4960 (2015).

[4] A. Bordonalli et al., "Optical Injection Locking to Optical Frequency Combs for Superchannel Coherent Detection," Opt. Exp. 23, p. 1547 (2015).

[5] Z. Tong et al., "Spectral Linewidth Preservation in Parametric Frequency Combs Seeded by Dual Pumps," Opt. Expr., vol 20, p. 17610 (2012). 